\title{
JCEED: A Joint Channel Assignment and Routing Protocol for Minimizing Delay in MR-MC Wireless Networks
}

\author{
Lei Zhou, Xianghui Cao, Yu Cheng and Hongkun Li \\ Department of Electrical and Computer Engineering, Illinois Institute of Technology, Chicago, IL 60616, USA \\ Email: lzhou17@hawk.iit.edu; \{xcao10,cheng\}@iit.edu; hli55@hawk.iit.edu
}

\begin{abstract}
Next generation wireless networks are widely based on the multi-radio multi-channel (MR-MC) networking model. While many existing works focus on network capacity and have shown that the capacity can be dramatically improved by using the MR-MC technique, the problem of delay-aware multi-hop routing in MR-MC networks has not been fully addressed. The problem mainly lies in the coupling between path selection and channel assignment. In this paper, we solve this problem by proposing a joint channel assignment and routing protocol based on end-to-end delay (JCEED) for MR-MC networks, where both transmission delay and queuing delay are taken into account. The protocol runs iteratively and in each iteration the channel assignment and path selection are updated based on previous iteration's performance in terms of end-to-end delay (EED). It finally finds the path which is both feasible and has minimum delay. Simulation results demonstrate the efficiency of the proposed JCEED protocol.
\end{abstract}

Index Terms-Multi-radio multi-channel networks; channel assignment; routing; end-to-end delay; joint design

\section{INTRODUCTION}

In multi-radio multi-channel (MR-MC) wireless networks, devices are equipped with multiple radios that can operate over multiple orthogonal channels. Such networking mode underpins the next generation wireless networks such as wireless mesh networks (WMN) based on the IEEE 802.16 standard, cognitive radio networks based on dynamic spectrum sharing, etc. [17]. The MR-MC networks have been shown efficient in improving network capacity [11], [14]. Due to the substantially increased system complexity, a great volume of literature has been devoted to analyzing throughput performance and designing efficient algorithms to achieve satisfactory throughput [1], [2], [15], [16].

The multi-hop routing is a critical issue in MR-MC networks. Existing studies in this direction mainly focus on throughput performance [1], [2], [15], where a widely adopted methodology is to minimize interference when selecting paths. For example, a joint channel assignment and routing protocol is proposed to select routes based on two linear programming based optimizations aiming at maximizing network throughput while minimizing total interference [9].

In many practical wireless networks such as VoIP and online gaming, there are strict delay requirements which make the routing problem challenging [9], [10]. Delay-aware multihop routing in single-radio single-channel (SR-SC) wireless networks have gained intensive research attention in the past decade [20]. For example, Gao et al. propose a routing protocol to achieve load balancing with minimum delay. Among these protocols, the routing metrics play an important role. The expected transmission count (ETX) describing the channel contention intension over a wireless link is adopted as the metric for designing routing [2]. This metric has been augmented by incorporating the impact of link positions. While these approaches are efficient for SR-SC networks, they do not directly apply to MR-MC networks with complex inter/intra- flow interferences [3].

Usually, the total delay of a network packet being served consists of transmission delay and queuing delay at the medium access control (MAC) layer. Some existing works have studied the delay-aware routing in the context of MRMC networks focusing on the transmission delay (e.g., [10]). However, in many real cases the queuing delay at the MAC layer takes an non-negligible portion of the total delay. Our previous work proposes the expected end-to-end delay (EED) metric which takes both transmission delay and queuing delay into account [3]. Integrating transmission delay with queuing delay, the EED metric can better account for network interferences and load-balancing. For example, in order to avoid large queuing delay, traffic may detour those congested links which can better balance the link loads [13].

In the context of MR-MC wireless mesh networks, there are also other routing metrics proposed in the literature. For example, the ETX metric is enhanced to link expected transmission time (ETT), and further associated with path weighted cumulative ETT (WCETT) [10]. While capturing intra-flow interferences, the ETT metric does not consider inter-flow interferences. In [3], a weighted expected end-toend delay (WEED) is proposed that takes into account both intra- and inter- flow interferences. WEED is a combination of the EED metric and a new path metric called multi-radio achievable bandwidth (MRAB). MRAB is intended to describe the effects of inter-flow and intra-flow interference [5], [7]. WEED based routing aims to obtain a path with minimized end-to-end delay under a given channel assignment, while minimizing co-channel interference.

In this paper, we investigate the multi-hop routing in MRMC wireless mesh networks taking both transmission delay and queuing delay into account. We aim to minimize the delay performance in terms of the EED while preserving high net- 
work throughput. A routing protocol that minimizes the EED based on presumed channel assignment has been proposed in [3]. However, unlike conventional SR-SC networks, MR-MC networks introduce complex coupling among path selection and channel assignment, making unilateral optimization inefficient in general MR-MC networks. In this paper, we jointly design routing and channel assignment and propose the joint channel assignment and routing protocol based on end-to-end delay (JCEED).

We use EED as a feedback information to recursively adjust the routing and channel assignment. In each updating, a path with minimum delay is selected, based on which the channel assignment is adjusted by scheduling those nodes on the selected path with better channels. To determine which channel is most suitable for a node on the selected path, we introduce a metric called channel interference index IDX [6], which can indicate the co-channel interference effect. Such a way can also benefit us in decreasing the interference and improving the network throughput. The JCEED protocol can finally find a path that minimizes the expected end-to-end delay while still achieving good throughput. We extend the ad-hoc on demand distance vector (AODV) protocol to implement our EED based routing. We let nodes send hello messages to their neighbors to obtain the information such as queue lengths and link qualities. Such information is then utilized to measure the probability of successful transmission and channel interference index.

Our major contributions can be summarized as follows.

- We take both transmission delay and queueing delay into consideration when capturing end-to-end delay.

- We propose a joint channel assignment and routing protocol for EED minimization in MR-MC networks. The protocol runs iteratively and in each iteration we use the the previous delay performance as feedback information to update the channel assignment accordingly.

- We conduct extensive simulations to demonstrate the efficiency of the proposed protocol.

The rest of this paper is organized as follows. Section II describes the system model. Section III presents the routing procedures under a given channel assignment. In Section IV, we give the detail design of the joint channel assignment and routing protocol. Section V shows the simulation results. Finally, Section VI concludes this paper.

\section{SySTEM MODEL}

We consider a general MR-MC network whose nodes form a directed graph $G(\mathcal{N}, \mathcal{L})$ with node set $\mathcal{N}$ and physical link set $\mathcal{L}$. A node $u$ is equipped with $\mathcal{R}_{u}$ radio interfaces such that two neighboring nodes can communicate with each other through multiple links at different channels simultaneously. The whole spectrum available to the network is divided into $\mathcal{C}$ orthogonal frequency channels. A link can be viewed as an allocation of a radio pair with one from the sender and the other from the receiver. There are two sources of conflicts that may cause failure to simultaneous transmissions over two links: the co-channel interference such that one link locates within the other's interference range; the radio conflict such that transmissions (possibly over different channels) contend for the same radio (which is the special issue in MR-MC networks). We assume that the MAC layer applies a carrier sensing multiple access (CSMA) based protocol to alleviate the co-channel interferences.

Consider that there are multiple flows traversing the network. Because of the above conflicts, there are both intra- and inter- flow interferences that can impact the end-to-end delay. Packets that are waiting for transmission through link $i$ are buffered in the MAC layer associated to the sender's radio of link $i$. Denote $Q_{i}$ as the number of packets buffered (or queue length) for link $i$.

An example MR-MC network is shown in Fig. 1. There is one commodity flow from the source $\mathrm{S}$ to its destination D. A, B, E and F are intermediate nodes. The variable $\mathrm{Q}$ denotes the number of packets buffered in each radio, $p$ is the probability of successful transmission over the link, and ch\# denotes the corresponding channel on which the links operate. For example, A uses its two radios to communicate with two radios of $\mathrm{B}$ via channel 1 and 2 , and the queue length for radio 1 of $\mathrm{A}$ is 5 packets and this radio has probability 0.9 to successfully transmit a packet to the corresponding receiving radio of $\mathrm{B}$. With $p$, we can estimate that on average it takes $\frac{1}{p}$ attempts to accomplish a successful transmission.

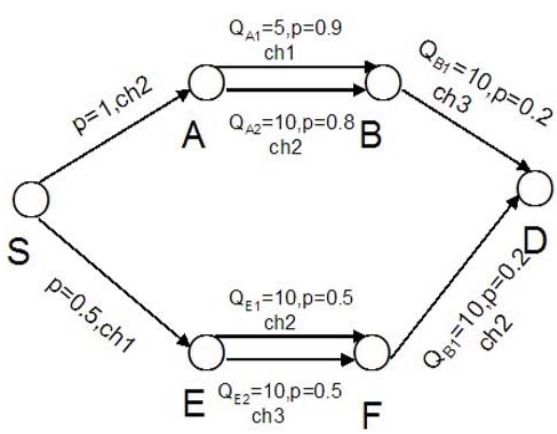

Fig. 1. An example MR-MC network.

\section{Minimum-Delay Routing Under Given Channel ASSIGNMENT}

In this section, we introduce the routing protocol that minimizes end-to-end delay under a given network channel assignment.

\section{A. Routing Metric}

Denote the transmission delay over link $i$ as $T_{i}$, and the queuing delay over this link as $Y_{i}$. Then the average delay for a packet to be transmitted over link $i$ is

$$
D_{i}=E\left[T_{i}+Y_{i}\right]
$$

Specifically, with the CSMA protocol, the transmission delay consists of not only the backoff delay but also the channel busy time due to transmissions from other nodes. Let $T_{i, n}$ denote the service time for the $n$-th samples over link $i$. 
With exponential weighed moving average scheme [12], the average transmission delay over link $i$ can be estimated as

$$
E\left[T_{i}\right]=(1-\beta) E\left[T_{i-1}\right]+\beta T_{i, n}, \quad 0 \leq \beta \leq 1
$$

Thus, if there are $\mathrm{Q}_{i}$ packets in the buffer when a new packet enters the queue of link $i$, the average delay over link $i$ can be estimated as

$$
D_{i}=\left(Q_{i}+1\right) E\left[T_{i}\right]
$$

where $Q_{i} * E\left[T_{i}\right]$ represents the queuing ahead of the new packet over link $i$ and $E\left[T_{i}\right]$ is the MAC service time of the new packet itself. Consider there are $H$ hops between source and destination, the EED of the path can be written as

$$
E E D=\sum_{i=1}^{H} D_{i}
$$

\section{B. Routing Protocol}

The routing protocol used in this paper is a variation of the one proposed in our previous work [3]. The major difference is that we use EED as our routing metric here while the weighted end-to-end delay (WEED) is adopted in [3]. WEED is a weighted combination of general EED and the multi-radio achievable bandwidth (MRAB) which is used to capture the effects of inter-flow and intra-flow interference. The key point of MRAB is to use an effective bandwidth to represent the actual bandwidth over a path. Although WEED explicitly expresses the effect of co-channel interference in the multi-hop wireless network environment, it's a subtle issue to determine the coefficient that reflects the relationship between delay and interference. In this paper, we let nodes send hello messages to neighbors to measure the probability of successful transmission between each other; the probabilities are further utilized to compute the transmission delay and queuing delay. In this way, the impact of intra- and inter- flow interference has been already reflected in the factors such as probability of successful transmission, which justifies the application of the EED metric.

Our routing protocol is an extension of the basic ad-hoc on demand distance vector (AODV) protocol to MR-MC networks with EED as the routing metric, where we assume that the channel assignment has been given beforehand. A (node, radio) tuple can be viewed as an independent entity in routing and information exchanging.

There are three types of control messages in our protocol: Route Request (RREQ), Route Reply (RREP) and Hello message. The source broadcasts an RREQ message to its neighbors via all of its radios. The RREQ message contains the information of the source tuple and a broadcast sequence number. Each tuple maintains a broadcast ID and increases it by 1 for each new RREQ. When a tuple receives an RREQ, it checks whether the RREQ has been received before by verifying the sequence number. If it is a new RREQ, the tuple will forward it; otherwise it will discard the RREQ automatically. Such procedures continue until the RREQ reaches the destination.
After receiving RREQ, an RREP packet will go back through the path the RREQ traverses from the destination, just in the reverse direction. Unlike RREQ, the RREP is applied with unicast transmissions over the path we select. Each RREQ or RREP message contains an EED field which stores the EED value of the path. When a fresh RREQ is received by a tuple, the tuple searches its neighbor list for the information of queue length and probability of successful transmission $p$, so as to calculate the EED value over the link between the previous tuple and the receiving tuple. Thus the cumulative EED over the sub-path until now can be updated by adding the new delay value. The EED of a path between source and destination can be obtained and with the EED metric tuples can update the routing table. In the routing table of the destination, the path with the smallest EED value is kept and now we find a path to minimize end-to-end delay.

The HELLO messages are employed to exchange the information between a tuple and its neighbors such as the queue length and link quality. This information is then utilized to estimate probabilities of successful transmission and channel interference indexes. Details will be described later in Section IV. Briefly, with the information obtained, tuples independently calculate the values of the routing metric to find a better path, and use the channel interference indexes to find a better channel.

\section{The Proposed Protocol: JCEED}

In this section, we present the detailed design of our joint routing and channel assignment protocol based on EED (JCEED) for MR-MC networks. In JCEED, each tuple exchanges information with its neighbors, estimates the successful transmission probabilities of its incident links and manages a routing table by calculating the EED metric of the sub-path between the source and itself.

For any given channel assignment, we can apply the above routing protocol to obtain a path with minimized end-to-end delay. However, we cannot ensure this channel assignment is optimal for the whole network. The basic idea of JCEED is to run the routing protocol iteratively to dynamically assign channels and select paths. It starts with an initial channel assignment (which can be achieved by randomly assign available channels) and processes with the routing protocol under this assignment. We introduce a term called channel interference index IDX [6], to represent how much co-channel interference caused by other tuples to the tuple under consideration when they share one channel. Specifically, for channel $i$, the interference index is

$$
I D X_{i}=\sum_{j=1}^{k} \frac{u \operatorname{sage}(i, j)}{j^{\gamma}}
$$

where usage $(i, j)$ denotes the number of packets sent by tuples using channel $i$ at the $j$-th hop from the tuple under consideration, $\gamma$ is the path loss exponent [19] and $k$ is the interference range.

Each tuple estimates the amount of data it sends and calculates IDX. In each iteration, after a path is selected via the 
EED based routing protocol, each tuple on this path chooses a channel with the smallest IDX, breaking the tie when there are multiple channels with the same smallest IDX by randomly selecting one. Thus, the alteration of channel assignment over this path changes the previous channel assignment into a new one. Based on the new channel assignment, we apply the routing protocol again to find a new path with minimized endto-end delay. We run this joint routing and channel assignment process iteratively until the result converges. Pseudo codes for the protocol are shown in the following algorithm.

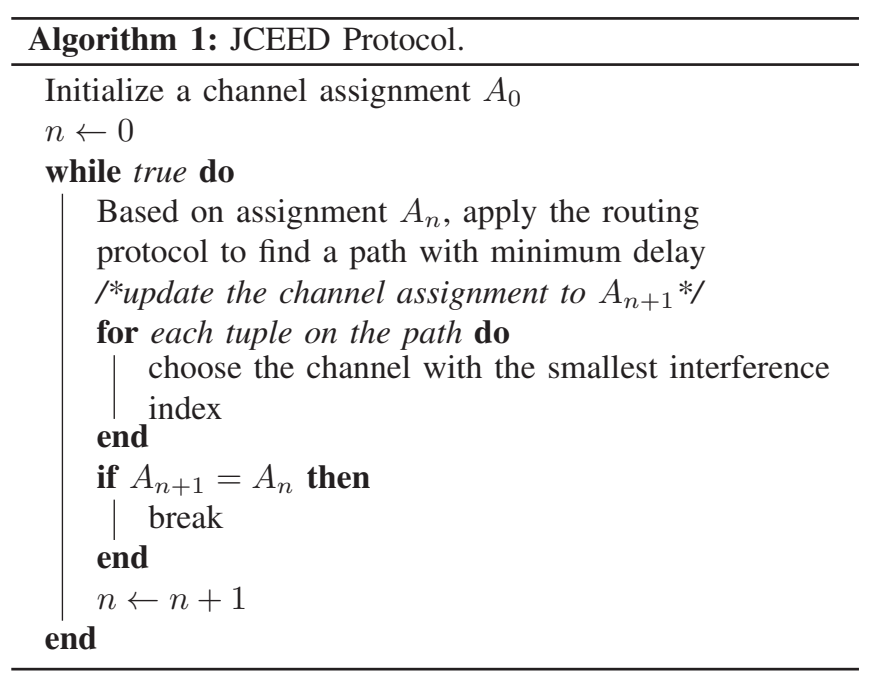

\section{Simulation RESUltS}

In this section we compare the performance of the proposed JCEED protocol with that of two existing protocols, i.e., the WCETT based protocol [10] and the WEED based protocol [3]. We develop our simulation program within the discrete event simulator NS2 [18]. We focus on an MR-MC wireless mesh network with 40 nodes which are randomly deployed in a $1000 m \times 1000 m$ square area. The network topology is shown in Fig. 2(a). Each node is equipped with 1 or 2 radios and each radio can communicate with its neighbors over 3 orthogonal channels. The transmission and interference range of each node is $250 \mathrm{~m}$ and $550 \mathrm{~m}$, respectively. There are 4 multi-hop flows over the network, and the source and destination nodes are denoted as $S_{i}$ and $D_{i}$, where $i=1,2,3,4$. Our aim is to find a path which has the minimized end-to-end delay between $S_{1}$ and $D_{1}$. Each node runs the IEEE 802.11 protocol, where the bandwidth of each channel is $11 \mathrm{Mbps}$ and the packet payload is 1000-bytes long.

Fig. 2(a) shows that with an initial channel assignment channel assignment, we can obtain a path through our routing algorithm with the smallest end-to-end delay. As shown in Fig. 2(a), the source of Flow 1, Node 9, routes to the destination Node 13 via Node 37, 18, 11, 25 over Channel $1,1,2,1$ and 3 , respectively. We can see that Channel 1 has been used three times over the path which could cause large intra-flow interference. Based on the proposed JCEED protocol, each node over the path turns to choose the channel with the smallest channel interference indexes. We can see from Fig. 2(b), in the final channel assignment, the source node finds a new path to reach the destination. The new path contains also 5 hops, but the hops use Channel 3, 1, 2, 3 and 1 , respectively, and the interference is thus reduced.

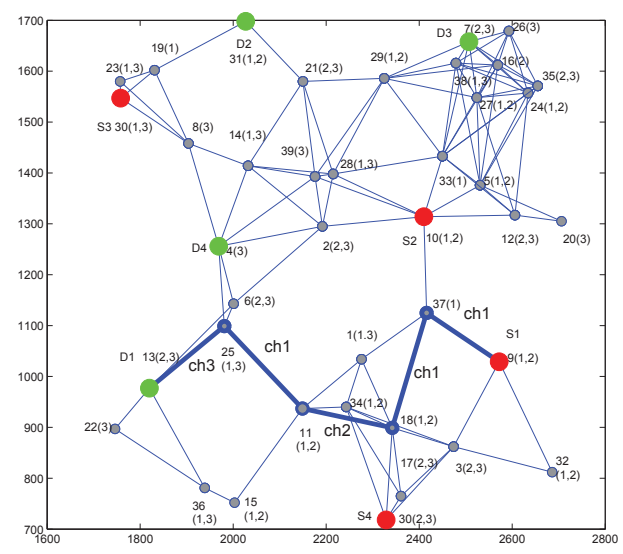

(a) Initial channel assignment

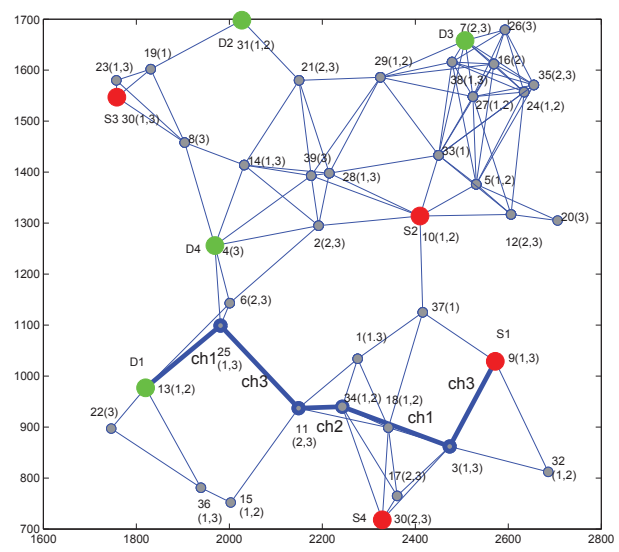

(b) Final channel assignment

Fig. 2. Snapshots of the network channel assignment. The texts close the nodes indicate radio-channel allocation. For example, 23(1,3) means that Node 23 has two radio interfaces and they are assigned to channel 1 and channel 3 respectively .

The performance comparison among WCETT, WEED and JCEED is shown in Fig. 3, from which we can see that the JCEED protocol outperforms the other two in general. From Fig. 3(a), we can see when heavy traffic is loaded to the network (e.g., per-flow rate larger than $0.6 \mathrm{Mbps}$ ), the throughput increases at a slow speed because the limited queue length cannot allow all the incoming packets to enter the queue, and thus packet loss takes place more frequently than the scenario with light traffic. The JCEED protocol shows better performance because nodes try to update for a least occupied channel during each iteration and thus lead the traffic to lightly loaded paths.

We can also see from Fig. 3(b) that the end-to-end delay 
with our protocol is smaller than those from the other two methods. When the flow rate is low, there is very low delay except for the transmission delay. When the flow rate rises, the channel becomes congested and end-to-end delay increases. However, the end-to-end delay becomes steady when flow rate is sufficiently high, because dropped packets are not accounted in delay and higher flow rate incurs higher dropping probability. Since our protocol seeks to redirect traffic to lightly loaded paths, the packet loss ratio decreases, which is clearly shown in Fig. 3(c).

\section{CONCLUSION}

In this paper, we have considered the routing problem in MR-MC networks by taking both the queuing delay and transmission delay into account. We propose a joint routing and channel assignment protocol (JCEED) that used the EED as the delay metric. JCEED runs iteratively and in each iteration the channel assignment and routing paths are adjusted based on the delay feedback information. Extensive NS2 simulation results demonstrate that the proposed JCEED protocol outperforms existing ones in terms of packet loss ratio, endto-end delay and throughput.

\section{REFERENCES}

[1] J. So and N. H. Vaidya, "Load-balancing routing in multichannel hybrid wireless networks with single network interface," IEEE Trans. Vehicular Technology, vol. 56, no. 1, pp. 342-348, Jan. 2007.

[2] D. S. J. De Couto, D. Aguayo, J. Bicket, and R. Morris, "A high throughput path metric for multi-hop wireless routing," in Proc. ACM MobiCom, pp. 134-142, 2003.

[3] H. Li, Y, Cheng, C. Zhou, and W. Zhuang, "Minimizing end-to-end delay: a novel routing metric for multi-radio wireless mesh networks," in Proc. IEEE INFOCOM, pp. 46-54, 2009.

[4] H. Li, Y. Cheng, and C. Zhou, "Multi-hop effective bandwidth based routing in multi-radio wireless mesh networks," in Proc. IEEE Globecom, pp 1-5, 2008.

[5] H. Li, Y. Cheng, C. Zhou, and W. Zhuang, "Routing metrics for minimizing end-to-end delay in multi-radio multi-channel wireless networks," IEEE Trans. Parallel and Distributed Systems, to appear,

[6] H. Chiu, K. Yeung, and K. Lui, "J-CAR: an efficient joint channel assignment and routing protocol for IEEE 802.11-based multi-channel multi-interface mobile ad hoc networks," IEEE Trans. wireless communications, vol. 8, no. 4, pp. 1706-1715, 2009

[7] K. Jain, J. Padhye, V. N. Padmanabhan, and L. Qiu, "Impact of interference on multi-hop wireless network performance," in Proc. ACM MobiCom, pp. 66-80, 2003.

[8] H. Zhai, J. Wang, and Y. Fang, "An efficient message authentication scheme for vehicular communications," in Proc. IEEE WCNC, pp. 10811086, 2004.

[9] M. Alicherry, R. Bhatia, and L. Li, "Joint channel assignment and routing for throughput optimization in multi-radio wireless mesh networks," in Proc. ACM MobiCom, pp. 114-128, 2004.

[10] R. Draves, J. Padhye, and B. Zill, "Routing in multi-radio, multi-hop wireless mesh networks," in Proc. ACM MobiCom, pp. 114-128, 2004.

[11] H. Li, Y. Cheng, C. Zhou, and P. Wan, "Multi-dimensional conflict graph based computing for optimal capacity in MR-MC wireless networks,", in Proc. IEEE ICDCS, Genoa, Italy, Jun. 2010.

[12] J. M. Lucas and M. S. Saccucci, "Exponentially weighted moving average control schemes: properties and enhancements," Technometrics, vol. 32 , no. 1 , pp. 1-12, 1990.

[13] J. Gao and L. Zhang, "Load-balanced short-path routing in wireless networks," IEEE Trans. Parallel and Distributed Systems, vol. 17, no.4, pp. 377-388, 2006.

[14] M. Kodialam and T. Nandagopal, "Characterizing the capacity region in multi-radio multi-channel wireless mesh networks," in Proc. ACM MobiCom, pp. 73-87, Aug. 2005.

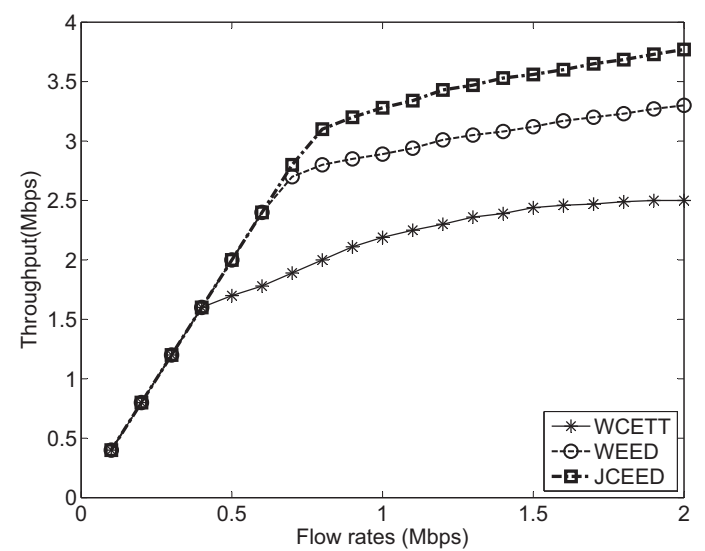

(a) Total throughput

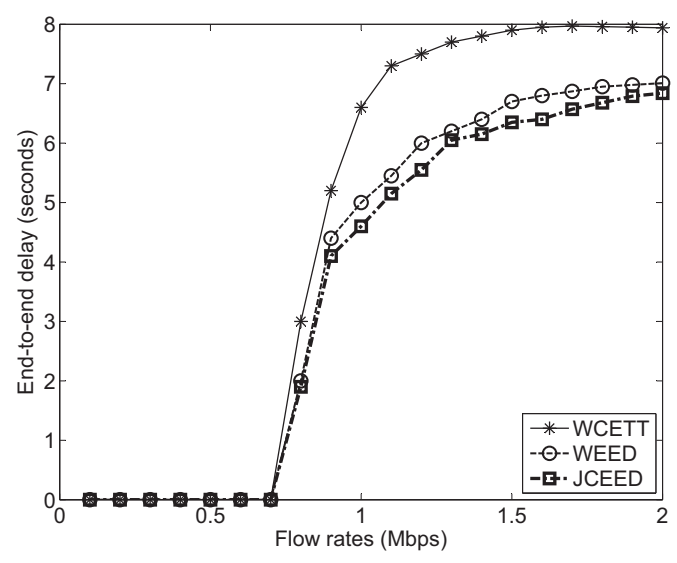

(b) End-to-end delay

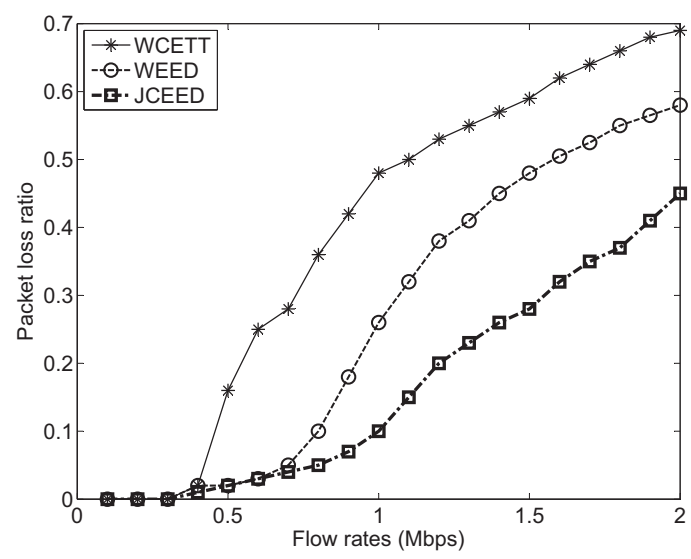

(c) Packet loss ratio

Fig. 3. Performance comparisons.

[15] A. Raniwala and T.-C. Chiueh, "Architecture and algorithms for an IEEE 802.11-based multi-channel wireless mesh network," in Proc.IEEE INFOCOM, pp. 2223-2234, 2005.

[16] T. Kuang, Q. Wu, and C. Williamson, "MRMC: a multi-rate multichannel MAC protocol for multi-radio wireless LANs," in Workshop on Wireless Networks and Communication Systems (WiNCS), pp. 263-272, Philadelphia, PA, Jul. 2005.

[17] Y. Cheng, H. Li, and P. Wan, "A theoretical framework for optimal 
cooperative networking in multi-radio multi-channel wireless networks," IEEE Wireless Communications Magazine, vol. 19, no. 2, pp. 66-73, 2012.

[18] R. A. Calvo and J. P. Campo, "Adding multiple interface support in NS-2," http://personales.unican.es/aguerocr/. 2007.

[19] G. Mao, B. D. O. Anderson, and B. Fidan, "Path loss exponent estimation for wireless sensor network localization," Computer Networks, vol. 51, no. 10 , pp. 421-436, 2006.

[20] M. E. M. Campista, P. M. Esposito, I. M. Moraes, L. H. M. Costa, O.

C. M. Duarte, D. G. Passos, C. V. N. de Albuquerque, D. C. M. Saade, and M. G. Rubinstein, "Routing metrics and protocols for wireless mesh networks.” IEEE Network, vol. 22, no. 1, pp 6-12, 2008. 